\title{
O USO DE NUTRIÇÃO ENTERAL PRECOCE PÓS-TRANSPLANTE HEPÁTICO ADULTO
}

\author{
Mário Reis ÁLVARES-da-SILVA, Catarina Bertaso Andreatta GOTTSCHALL, \\ Fábio Luis WAECHTER, Everton HADLICH, José Arthur SAMPAIO e \\ Carlos Fernando M. FRANCESCONI
}

RESUMO - Racional - O transplante hepático é o tratamento de escolha para várias doenças hepáticas terminais. A desnutrição nesta população é freqüente. $\mathrm{O}$ uso de nutrição enteral precoce não é rotina nos grupos de transplante. Objetivo - Relatar a experiência do uso de nutrição enteral precoce em indivíduos transplantados hepáticos e avaliar sua segurança. Pacientes/Métodos - Foram estudados 35 adultos submetidos a transplante hepático. A avaliação do estado nutricional pré-transplante hepático foi realizada pela avaliação nutricional subjetiva global e força do aperto da mão não-dominante, aferida pela dinamometria. A dieta enteral foi iniciada em até 12 horas. A via oral foi iniciada assim que houvesse ruídos hidroaéreos. Resultados - A média de idade dos indivíduos avaliados foi de 45,5 anos ( \pm 8,93). De acordo com a avaliação nutricional subjetiva global, a prevalência de desnutrição pré-transplante foi de $77,1 \%$ e pela força do aperto da mão não-dominante de $100 \%$. A nutrição enteral precoce foi iniciada em até 12 horas, moda de 10,9 horas e mantida exclusivamente por período médio de 2,6 dias $( \pm 2,2)$. Via oral exclusiva foi obtida no período médio de 9,5 dias $( \pm 9,7)$. A nutrição enteral precoce proveu o aporte calórico estimado em $97 \%$ dos casos. Intolerância à dieta enteral ocorreu em cinco indivíduos (14,2\%), sendo que em quatro foi reinstituída com sucesso após intervalo de 12 horas. A prevalência de infecção respiratória foi de 28,6\%. Em apenas dois pacientes $(5,7 \%)$ houve broncopneumonia com aspecto aspirativo ao estudo radiológico do tórax. Conclusães - A nutrição enteral precoce foi um método eficaz na provisão de calorias e seguro em sua aplicação a pacientes transplantados hepáticos.

DESCRITORES - Nutrição enteral. Transplante de fígado.

\section{INTRODUÇÃO}

O transplante hepático (TXH) é atualmente o tratamento de escolha para uma série de doenças terminais agudas e crônicas do fígado ${ }^{(2,3,8,14)}$. A maioria dos pacientes levados a transplante, no entanto, é desnutrida ${ }^{(16,21,23)}$ e isto pode contribuir para maior morbimortalidade pós-operatória, bem como maior custo ${ }^{(5,20)}$. Os pacientes com doença hepática grave têm risco aumentado de aquisição de infecções ${ }^{(5)}$, reconhecidamente a principal causa de óbito pós-transplante ${ }^{(2)}$. A translocação bacteriana, por sua vez, é responsável por parcela considerável desses eventos. A nutrição enteral (NE), por evitar atrofia da mucosa intestinal, reduz a translocação, podendo reduzir as complicações sépticas ${ }^{(23)}$. O uso de nutrição enteral precoce (NEP), no entanto, não é rotina disseminada nos grupos de transplante. Revisão publicada em nosso meio sugere que a alimentação seja iniciada a partir do terceiro ou quarto dia pós-TXH ${ }^{(19)}$. Ainda recentemente, foi publicado estudo japonês relatando quatro casos com o uso de NEP em transplante hepático ${ }^{(24)}$.

O objetivo deste estudo foi relatar a experiência obtida com o uso de NEP em indivíduos transplantados hepáticos e avaliar sua segurança.

\section{CASUÍSTICA E MÉTODOS}

Foi realizado estudo prospectivo em 41 indivíduos adultos com doença hepática crônica terminal, consecutivamente submetidos a TXH, num período de 52 meses no Hospital de Clínicas de Porto Alegre, RS. Foram excluídos da amostra os pacientes que se encontravam hospitalizados imediatamente antes do transplante, os casos de TXH de emergência por insuficiência hepática aguda grave e os de retransplante. Todos os indivíduos foram submetidos a imunossupressão tríplice com ciclosporina em microemulsão, prednisona e azatioprina. A avaliação do estado nutricional pré-TXH foi realizada através da avaliação nutricional subjetiva global (ANSG) e da força do aperto da mão não-dominante (FAM), aferida pela dinamometria ${ }^{(1,4,9,11,25)}$.

Durante a cirurgia foi instalada sonda nasoentérica (SNE) de $10 \mathrm{~F}$ em todos os indivíduos, posicionada pelo cirurgião, no duodeno. Logo após a chegada ao centro de tratamento intensivo (CTI), mesmo ainda sem ruídos hidroaéreos (RHA), foi iniciada administração de solução de água e glutamina por bomba de infusão. Em até 12 horas, esta solução foi substituída pela infusão contínua de dieta líquida, polimérica, sem lactose e sacarose, e com fibras, na velocidade inicial de $50 \mathrm{~mL} / \mathrm{h}$ e, conforme tolerância, 70 ou $80 \mathrm{~mL}$ e sucessivamente até 100

Grupo de Transplante Hepático do Hospital de Clínicas de Porto Alegre, RS

Endereço para correspondência: Nutr. Catarina Bertaso Andreatta Gottschall - Rua Des. Augusto Loureiro Lima, 129 - apto 402 - Bairro Petrópolis - $90470-120$ - Porto Alegre, RS. E-mail: cgottschall@hcpa.ufrgs.br 
$\mathrm{mL} / \mathrm{h}$. A partir do momento em que os RHA estivessem presentes, o paciente recebia também líquidos por via oral. De acordo com a evolução, a dieta líquida foi gradativamente substituída por dieta normal com controle de sódio (com as necessárias adequações individuais em relação ao conteúdo nutricional). No momento em que o paciente estivesse ingerindo dois terços de suas necessidades calóricas por via oral, estimadas pela equação de predição de HarrisBenedict, a SNE foi retirada. Durante todo o período, a aceitação da dieta enteral ou oral era monitorada em relação a efeitos adversos, como distensão abdominal, náuseas e vômitos. A presença de algum destes sintomas foi motivo de suspensão da dieta, com retomada após pausa de 12 horas.

No período pós-operatório inicial os pacientes eram controlados diariamente, até que fosse retirada a SNE, no que se refere aos seguintes itens: a) quantidade de calorias efetivamente ingeridas, b) tolerância à dieta e c) sinais de infecção, especialmente respiratória. Foram também considerados os dias de internação em CTI, o número de dias de intubação orotraqueal e a permanência hospitalar.

\section{RESULTADOS}

Dos 41 pacientes submetidos a TXH no período, 6 foram excluídos: 2 deles por óbito no transoperatório e os 4 restantes por dificuldades operacionais (não colocação da SNE no transoperatório em 1, e início inadvertido da solução enteral de água e glutamina 12 horas após o TXH em 3). Assim, 35 indivíduos foram avaliados, sendo que $24(68,6 \%)$ do sexo masculino e $11(28,6 \%)$ do feminino. A média de idade foi de 45,5 anos $( \pm 8,93)$.

De acordo com a ANSG, a prevalência de desnutrição pré-transplante foi de $77,1 \%$, enquanto que pela FAM $100 \%$ dos pacientes foram considerados desnutridos.

A NE foi iniciada em todos os indivíduos em até 12 horas, moda de 10,9 horas, e foi mantida com exclusividade por período médio de 2,6 dias $( \pm 2,2)$. Via oral exclusiva foi obtida no período médio de 9,5 dias $( \pm 9,7)$. A NEP proveu o aporte calórico estimado para o indivíduo em $97 \%$ dos casos.

O tempo médio de permanência na CTI foi de 8,2 dias $( \pm 5,3)$, tendo sido a extubação realizada, em média, em 2,3 dias $( \pm 2,9)$. Os pacientes permaneceram internados, em média, por 32 dias no hospital $( \pm 15,8)$.

Intolerância à dieta enteral ocorreu em cinco indivíduos (14,2\%), sendo que em quatro deles ela foi reinstituída com sucesso após intervalo de 12 horas. No paciente restante houve intolerância após a reinstituição, motivando a sua retirada.

A prevalência de infecção respiratória foi de $28,6 \%$. Em apenas dois pacientes $(5,7 \%)$ houve broncopneumonia com aspecto aspirativo ao estudo radiológico do tórax.

\section{DISCUSSÃO}

A DPC tem sido relacionada a maior morbimortalidade pósTXH. Na aferição da FAM, por exemplo, o desempenho abaixo de
$85 \%$ do esperado tem sido associado à incidência aumentada de infecção e maior permanência em $\operatorname{CTI}^{(7,13)}$. A população avaliada neste estudo era de indivíduos com alta prevalência de desnutrição, o que vai ao encontro dos achados de outras séries de pacientes em lista de espera para $\mathrm{TXH}^{(17)}$.

A intervenção nutricional nestes indivíduos pode ser realizada no período pré ou no pós-operatório. No período pré-TXH, a presença de doença hepática e seu conseqüente impacto sobre o estado nutricional muitas vezes não permite adequada correção. De fato, estudo randomizado aferindo o impacto da intervenção nutricional pré-TXH, demonstrou melhora dos parâmetros nutricionais pré-operatórios, incluindo ANSG e FAM, sem no entanto, demonstrar diferença significativa na sobrevida a curto prazo $^{(7)}$. A NEP poderia contribuir para a correção do estado nutricional no transplante imediato, com isto concorrendo para melhores resultados ${ }^{(10,12)}$. Ao menos em modelo animal, LEVY e ALEXANDER $^{(15)}$ demonstraram aumento na sobrevida de enxertos alogênicos cardíacos tratados com NE. Em humanos, a intervenção nutricional enteral em mulheres idosas desnutridas, submetidas a cirurgia ortopédica, relacionou-se a menores complicações e menor tempo de hospitalização ${ }^{(13)}$. Recentemente, RAYES et al..$^{(22)}$ demonstraram a utilidade de suplementação enteral de lactobacilos em pacientes submetidos a transplante hepático, em decorrência da menor translocação bacteriana.

Se alimentar precocemente o indivíduo transplantado parece interessante e justificável, a via oral nem sempre está disponível, quer seja por complicações clínicas (alterações do sensório são freqüentes no pós-operatório e os períodos de intubação orotraqueal são variados) ou ainda pela freqüente presença de anorexia nesta fase. A alternativa à alimentação enteral seria a nutrição parenteral total, cujo papel não está bem dimensionado nesta população ${ }^{(17,18)}$. Daí o porquê da eleição da nutrição enteral para trabalhar nesta amostra.

A tolerância à dieta enteral foi boa, as complicações diretamente dela advindas foram pouco prevalentes e solucionadas com a suspensão temporária da infusão. Ressalva-se, no entanto, a ocorrência de broncopneumonia aspirativa com evolução a óbito em um dos pacientes desta série, que havia demonstrado sinais de intolerância (vômitos) à dieta. No único paciente em que não foi possível reinstituir a nutrição enteral por intolerância, constatou-se piora clínica a seguir, tendo o paciente evoluído a óbito por grave disfunção do enxerto. A intolerância nutricional foi atribuída à falência intestinal associada à disfunção de múltiplos órgãos. Entretanto, o número de casos de infecção respiratória, efeito adverso teoricamente bastante relacionado à intolerância, especialmente a náuseas e vômitos, foi inferior ao relatado na literatura ${ }^{(6)}$.

\section{CONCLUSÕES}

Os achados deste estudo, mesmo considerando a pequena amostra, sugerem que seja a NEP método eficaz na provisão de calorias e seguro em sua aplicação a pacientes transplantados de fígado. 
Álvares-da-Silva MR, Gottschall CBA, Waechter FL, Hadlich E, Sampaio JA, Francesconi CFM. The use of early enteral feeding post orthotopic liver transplantation in adults. Arq Gastroenterol 2004;41(3):147-9.

ABSTRACT - Background - Orthotopic liver transplantation is the treatment of choice for end-stage liver disease. Malnutrition is common in this population. Early enteral nutrition is not routine in the transplant groups. Aim - To report our experience with the use of early enteral nutrition in patients undergoing orthotopic liver transplantation and also evaluate its safety. Patients/Methods - We studied 41 adults submitted to orthotopic liver transplantation. Pre- orthotopic liver transplantation nutritional assessment was accomplished by the subjective global assessment and grip strength. Enteral nutrition was begun in 12 hours. Oral feeding was initiate gradually as soon as possible. Results - We studied 35 individuals, with an average of age of 45.5 years (8.93). The prevalence of malnutrition in orthotopic liver transplantation was of $77.1 \%$ determined by subjective global assessment, and $100 \%$ by grip strength. Early enteral nutrition was begun in all of the individuals in up to 12 hours, mode 10.9 hours, and maintained exclusively by medium period of 2.6 days (2.2). Oral feeding was obtained in the medium period of 9.5 days (9.7). Early enteral nutrition provided total caloric intake in $97 \%$ of the cases. Intolerance to the enteral feeding occurred in five individuals (14.2\%), and in four of them it was resumed successfully after 12 hours. The prevalence of respiratory infection was of $28.6 \%$. In only two patients (5.7\%) there was aspirative bronchopneumonia. Conclusions - Early enteral nutrition is an effective method in the provision of calories and safe in application to patients undergoing orthotopic liver transplantation.

HEADINGS - Enteral nutrition. Liver transplantation.

\section{REFERÊNCIAS BIBLIOGRÁFICAS}

1. Álvares-da-Silva MR, Silveira TR. O estudo da força do aperto da mão não-dominante em indivíduos sadios. Determinação dos valores de referência para o uso da dinamometria. GED Gastroenterol Endosc Dig 1998;6203-6.

2. Charlton M. Natural history of hepatitis $\mathrm{C}$ and outcomes following liver transplantation. Clin Liver Dis 2003;7:585-602.

3. Crossin JD, Muradali D, Wilson SR. US of liver transplants: normal and abnormal. Radiographics 2003;23:1093-114.

4. Detsky AS, Baker JP, O’Rourke K, Johnston N, Whitwell J, Mendelson RA, Jeejeebhoy KN. Predicting nutrition-associated complications for patients undergoing gastrointestinal surgery. JPEN J Parenter Enteral Nutr 1987;11:440-6.

5. Driscoll DF, Palombo JD, Bistrian BR. Nutritional and metabolic considerations of liver transplant candidate and organ donor. Nutrition 1995;11:255-63.

6. Durán FG, Piqueras B, Romero M, Carneros JA, De Diego A, Salcedo M, Santos L, Ferreiroa J, Cos E, Clemente G. Pulmonary complications following orthotopic liver transplant. Tranpl Int 1998;11 Suppl 1 s255-s9.

7. Figueiredo F, Dickson ER, Pasha T, Kasparova P, Therneau T, Malinchoc M, DiCecco S, Francisco-Ziller N, Charlton M. Impact of nutritional status on outcome after liver transplantation. Transplantation 2000;70(9):1347-52.

8. Goff JS, Glazner J, Bilir, BM. Measuring outcome after liver transplantation: a critical review. Liver Transplant Surg 1996;4:189.

9. Gottschall CBA. Avaliação nutricional de pacientes com cirrose pelo vírus da hepatite C: a aplicação da calorimetria indireta [dissertação]. Porto Alegre: Curso de Pós-Graduação em Gastroenterologia da Universidade federal do Rio Grande do Sul; 2003.

10. Hasse JM, Blue LS, Liepa GU, Goldstein RM, Jennings LW, Mor E, Husberg BS, Levy MF, Gonwa TA, Klintmalm GB. Early enteral nutrition support in patients undergoing liver transplantation. JPEN J Parenter Enteral Nutr 1995; 19:437-43.

11. Humphreys MD, Maza P, Hirsch S, Barrera G, Gattas V, Bunout D. Muscle strength as a predictor of loss of functional status in hospitalizer patients. Nutrition 2002;18:616-20.

12. Klein S, Kinney J, Jeejeebhoy K, Alpers D, Hellerstein M, Murray M, Twomey P Nutrition support in clinical practice: review of published data and recommendations for research directions. National Institutes of Health, American Society for Parentera and Enteral Nutrition, and American Society for Clinical Nutrition. JPEN J Parenter Enteral Nutr 1997;21:133-56.
13. Le Cornu KA, McKierman FJ, Kapadia AS, Neuberger JM. A prospective randomized study of nutritional supplementation in patients awaiting elective orthotopic liver transplantation. Transplantation 2000;69:1364-69.

14. Lee WM. Acute liver failure in the United States. Semin Liver Dis 2003;23:217-26.

15. Levy AE, Alexander JW. Nutritional immunomodulation enhances cardiac allograft survival in rats treated with donor-specific transfusion and cyclosporine. Transplantation 1995;60:812-5.

16. Matos C, Poraykomk MK, Francisco-Ziller N, DiCecco S. Nutrition in chronic liver disease. J Clin Gastroenterol 2002;35:391-7.

17. McCullough AJ. Malnutrition in liver disease. Liver Transpl 2000;6: Suppl 1: s85-96.

18. O'Grady JG. Clinical economics review: liver transplantation. Alim Pharmacol Ther 1997; 11:445s-51s.

19. Parolin MB, Zaina FE, Lopes RW. Terapia nutricional no transplante hepático. Arq Gastroenterol 2002;39:114-22.

20. Pikul J, Sharpe MD, Lowdes R. Degree of preoperative malnutrition is predictive of postoperative morbitity and mortality in liver transplant recipients. Transplantation 1994;57:469-72.

21. Plevak DJ, DiCecco SR, Wiesner RH, Porayko MK, Wahlstrom HE, Janzow DJ, Hammel KD, O'Keefe SJ. Nutritional support for liver transplantation: identifying caloric and protein requeriments. Mayo Clin Proc 1994;69:225-30.

22. Rayes N, Seehofer D, Hansen S, Boucseink S, Müller AR, Serke S, Bengmark S, Neuhaus P. Early enteral supply of lactobacillus and fiber versus selective bowel decontamination: a controlled trial in liver transplant recipients. Transplantation 2002;74:123-7.

23. Riordan SM, Williams R. Nutritional and liver transplantation. J Hepatol 1999;31:955-62.

24. Sekido H, Matsuo K, Takeda K, Morioka D, Kubota T, Tanaka K, Endo I, Togo S, Tanaka K, Shimada H. Impact of early enteral nutrition after liver transplantation for acute hepatic failure: report of four cases. Transpl Proc 2003;35:369-71.

25. Stephenson RG, Moretti EW, El-Moalen H, Clavien PA, Tuttle-Newall JE. Malnutrition in liver transplant patients. Transplantation 2001;72:666-70.

Recebido em 1/8/2003. Aprovado em 28/11/2003. 